\title{
Life Expectancy in the Spouses of Veterans
}

\section{ART ICLE INF O}

\section{Article Type}

Descriptive Study

\section{Authors}

Samadifard H.R.* $M A$,

Narimani M. ${ }^{1} \mathrm{PhD}$

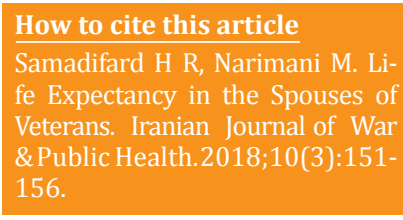

*Psychology Department, Educational Sciences \& Psychology Faculty, University of Mohaghegh Ardabili, Ardabil, Iran

${ }^{1}$ Psychology Department, Educational Sciences and Psychology Faculty, University of Mohaghegh Ardabili, Ardabil, Iran

\section{Correspondence}

Address: University of Mohaghegh Ardabili, Daneshgah Street, Aradabil, Iran. Postal Code: 56199-11367 Phone: +98 (45) 33510135

Fax: +98 (45) 33510135

hrsamadifard@ymail.com

\section{Article History}

Received: October 25, 2017

Accepted: March 06, 2018

ePublished: August 29, 2018

\section{A B S T R A C T}

Aims Spouses in the role of caring for veterans are under the influence of stresses and problems which threaten their health and their families' health. On the other hand, spouses with high life expectancy will have high quality of life satisfaction. The purpose of this study was to determine the role of perceived social support, mindfulness and cognitive flexibility in predicting life expectancies of spouses of veterans.

Instruments \& Methods The present study is a descriptive-correlational. The statistical population of the study includes all the spouses of veterans supported by Martyr and Veterans Affairs Foundation of Ardabil in 2016 (N=3400). Using available sampling, 110 of them were selected as sample. For data collection, perceived social support (MSPSS), mindfulness (MASS), cognitive flexibility (CFQ) and life expectancy scale (SHS) were used. Data were analyzed by Pearson correlation coefficient and multiple regressions using SPSS 23 software.

Findings There was a significant relationship between the perceived social support, mindfulness and cognitive flexibility with spouses' life expectancy $(\mathrm{p}<0.05)$. Also the perceived social support, mindfulness and cognitive flexibility could predict the life expectancy of spouses of veterans by $47 \%(\mathrm{p}<0.05)$.

Conclusion That the perceived social support, mindfulness and cognitive flexibility are included in the variables relevant to the life expectancy and they can predict it. In general, the spouses having higher levels of perceived social support, mindfulness and cognitive flexibility also have higher of life expectancy compare with other spouses.

Key words Social Support; Mindfulness; Cognition; Life Expectancy; Spouses; Veterans

\section{I T A T I O N L I N KS}

[1] Comparison of quality of life, depression, stress, anxiety... [2] Experiences of military spouses of veterans with ... [3] Quality of life of caregiver spouses of veterans ... [4] Amouchie. Secondary traumatic stress, dissociative ... [5] The role of coping, resilience, and social ... [6] The role of religious orientation and life expectancy ... [7] The role of hope, loneliness feeling and their components ... [8] Spousal mindfulness and social support in ... [9] The relationship between mindfulness and ... [10] You can't always get what you want, but can ... [11] Relationship, social support and personality as ... [12] Correlation of social support with social health ... [13] The relationship between social support and marital ... [14] Relationship between social support and quality of life dimensions ... [15] Partridge K. Letting go: Mindfulness and ... [16] Mindfulness, self-compassion and happiness in non-meditators ... [17] Emotional resilience mediates the relationship between ... [18] Mindfulness, self-compassion, posttraumatic stress ... [19] Are mindfulness and self-compassion ... [20] The effect of mindfulness-based cognitive ... [21] The effectiveness of mindfulness-based stress reduction ... [22] Predictingthe quality of marital relationship on ... [23] Demystifying cognitive flexibility: Implications for ... [24] The role of cognitive flexibility and mindfulness in predicting ... [25] Investigating cognitive flexibility as a potential ... [26] Correlates of cognitive flexibility in veterans ... [27] Explanation of psychological problems ... [28] The relationship between life's meaningfulness, hope ... [29] The role of hope as a mediator in recollected ... [30] The relationship of optimism and hope with depression ... [31] The multidimensional scale of perceived ... [32] Psychometric properties of the multidimensional scale ... [33] Internet and communication: Perceived social support ... [34] Why we don't need self-esteem: On fundamental ... [35] Mindfulness in Iran and the United States: Cross-cultural ... [36] The cognitive flexibility inventory: Instrument development ... [37] The mediating role of cognitive flexibility in correlation ... [38] The will and the ways: Development and ... [39] Prediction of life expectancy of spouses based of metacognitive ... 


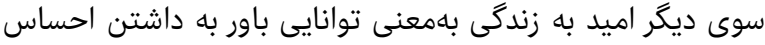

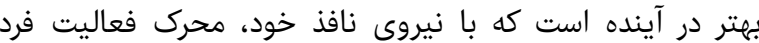

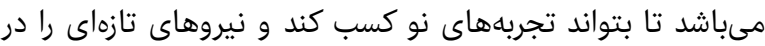

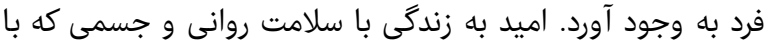

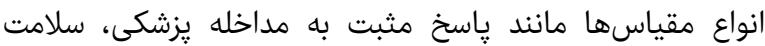

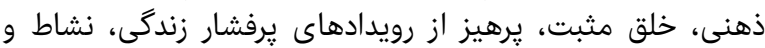

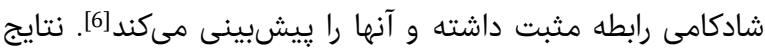

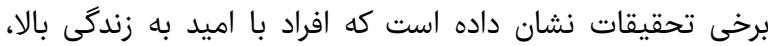

رضايت زندگى مطلوبى دارند]7].

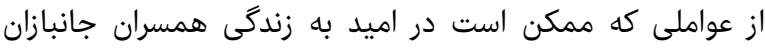

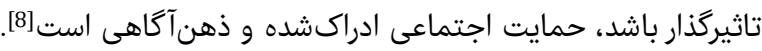

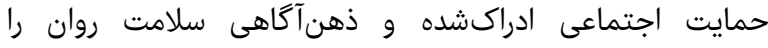

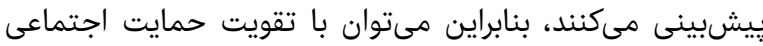

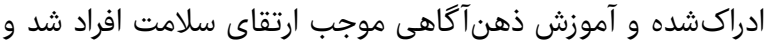

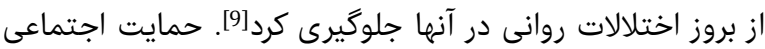

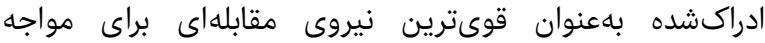

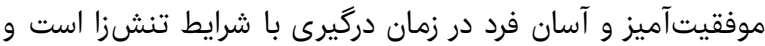

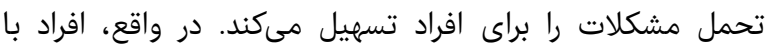

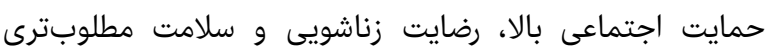

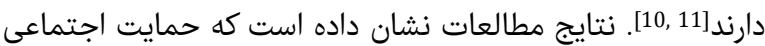

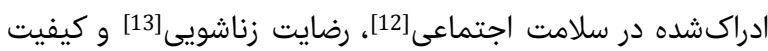

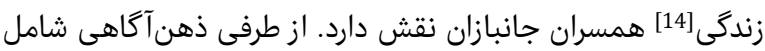

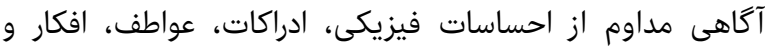

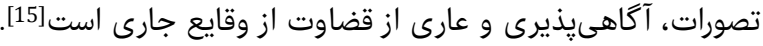

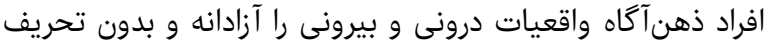

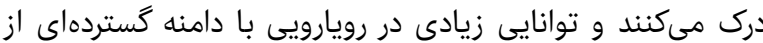

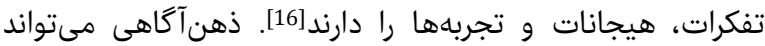

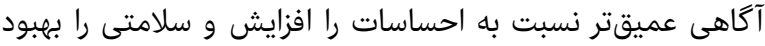

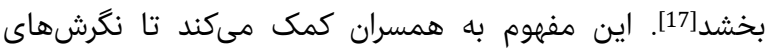

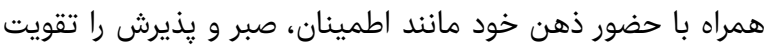

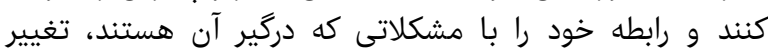

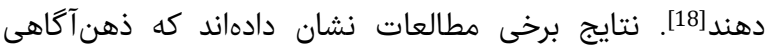

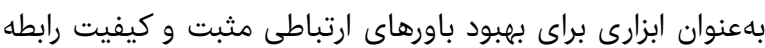

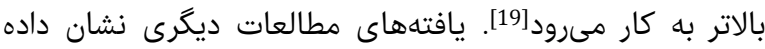

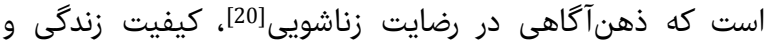

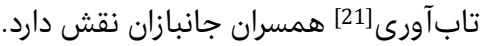

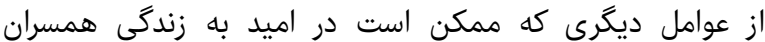

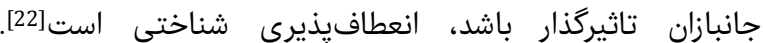

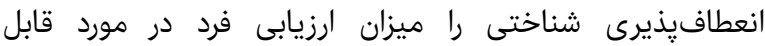

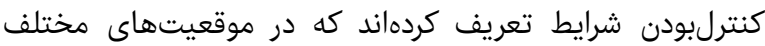

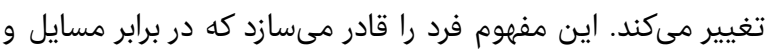

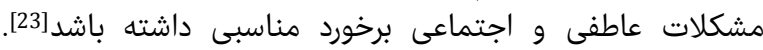

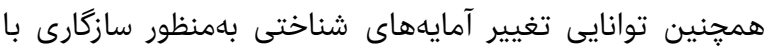

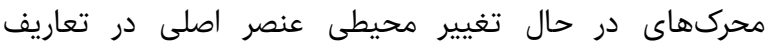

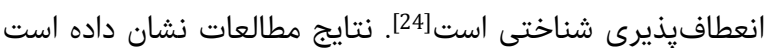

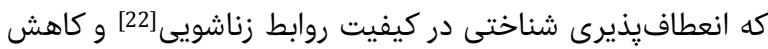

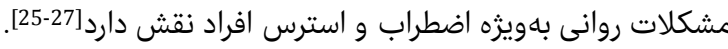

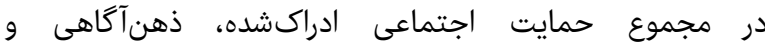

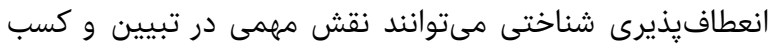

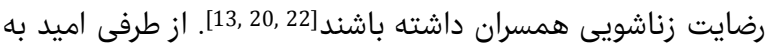

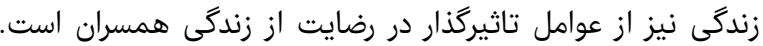

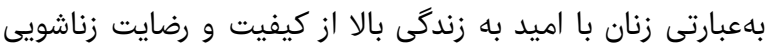

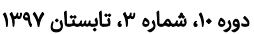

اميد به زندگى در همسران جانبازان

MA* حميدرضا صمدىفرود كروه روان شناسى، دانشكده علوم تربئه

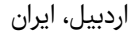

PhD محمد نريمانى ايران كروه روان شناسى، دانشكده علوم تربيتى و روانشناسى، دانشكاه محقق اردبيلى،

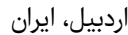

جكيده

أهداف: همسران در نقش مراقبتكننده از جانبازان، تحت تاثير استرسها و وانوان

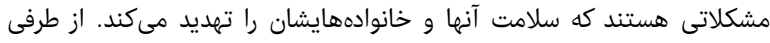

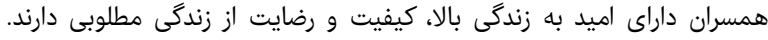

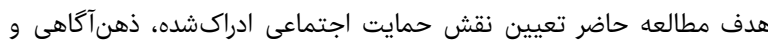

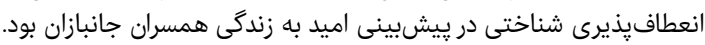

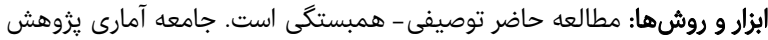

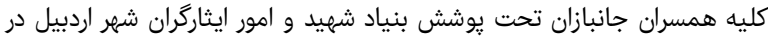

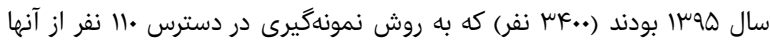

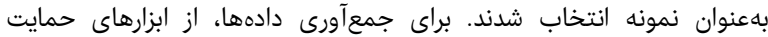

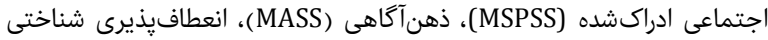

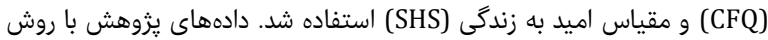

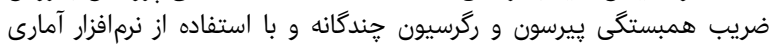
SPSS 23

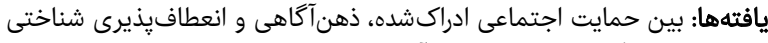

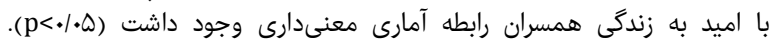

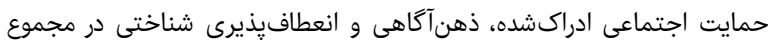

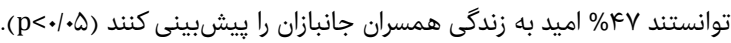

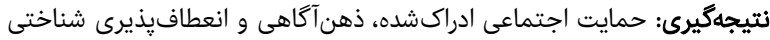

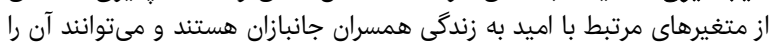

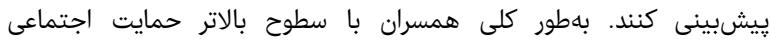

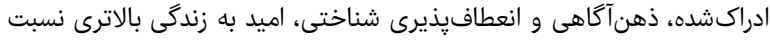

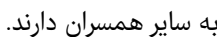
كليدواثهها: حمايت اجتماعى ادراكشده، ذهن آكاهي، انعطافيذيرى شناختى، اميد به

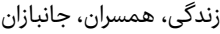

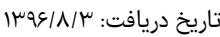

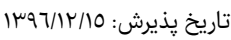

'نويسنده مسئول: hrsamadifard@ymail.com

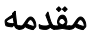

جنگ ايران و عراق و استرسهاى ناشى از آن موجب شده است كه

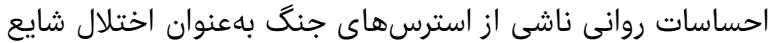

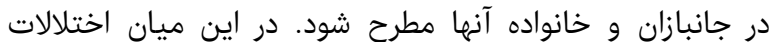

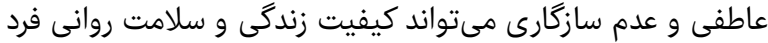

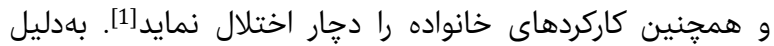

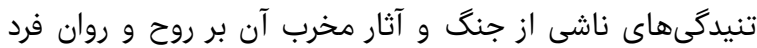

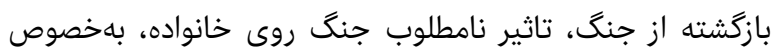

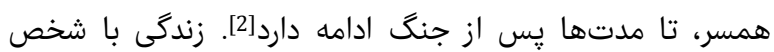

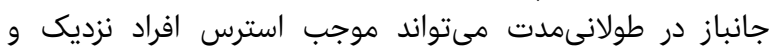

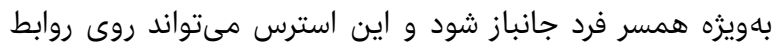

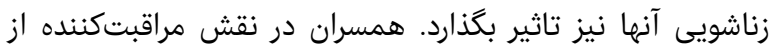

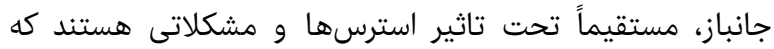

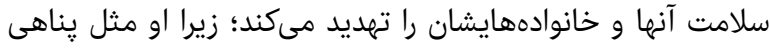

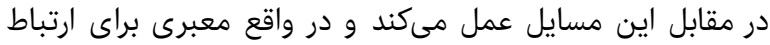

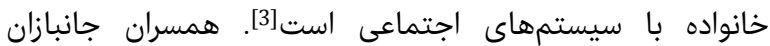

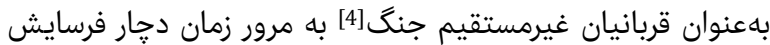

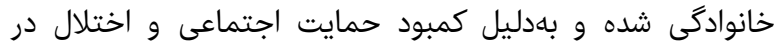

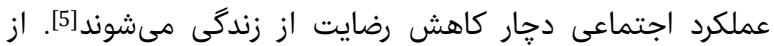




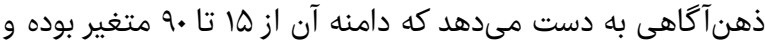

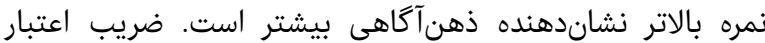

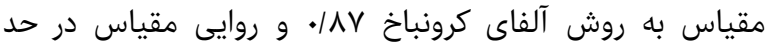

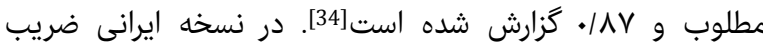

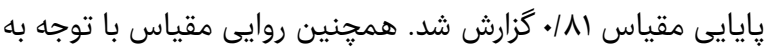

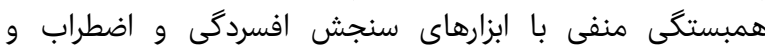

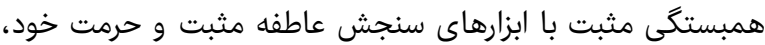

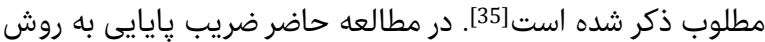

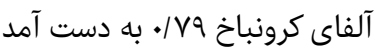

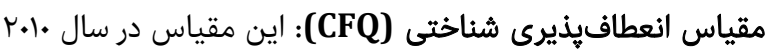

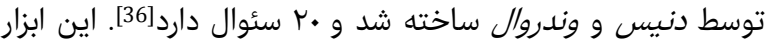

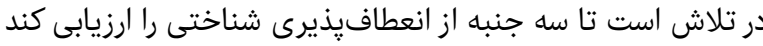

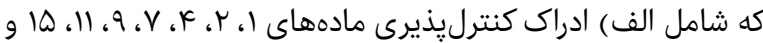

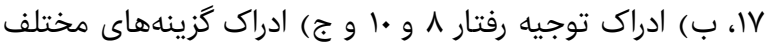

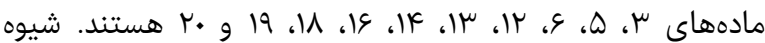

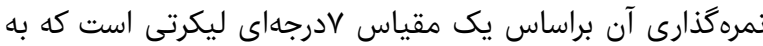

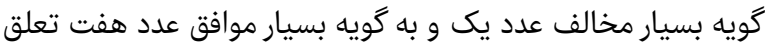

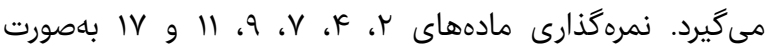

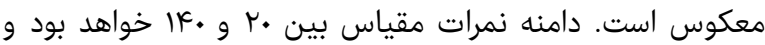

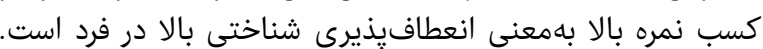

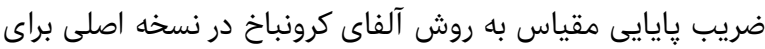

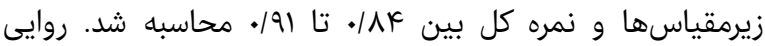

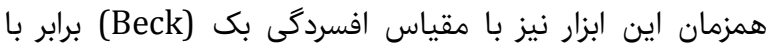

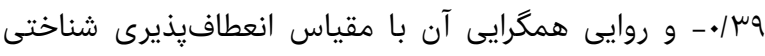

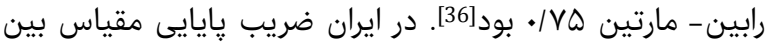

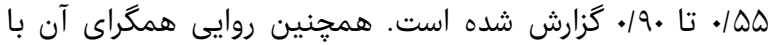

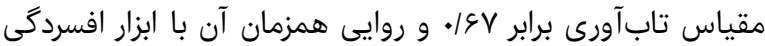

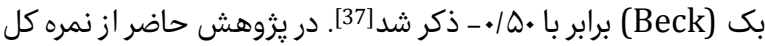

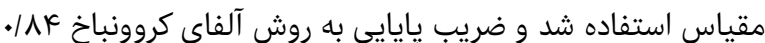

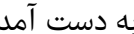
مقياس اميد به زندگى (SHS): اين ابزار توسط اسنايدر در سال

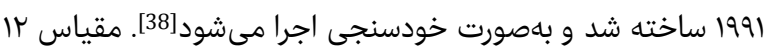

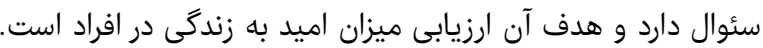

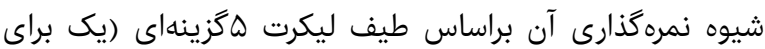

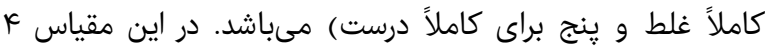

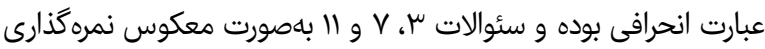

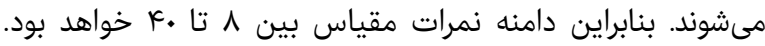

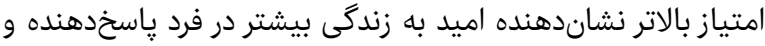

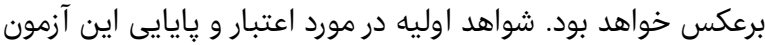

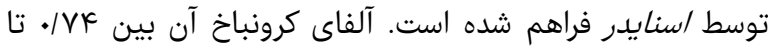

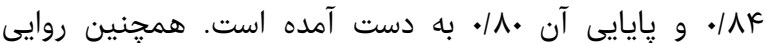

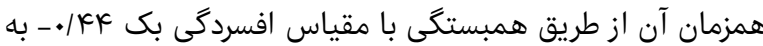

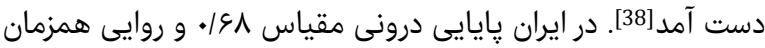

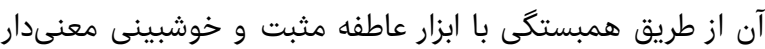

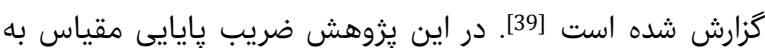

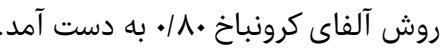

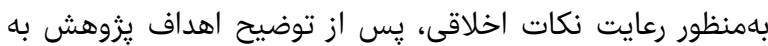

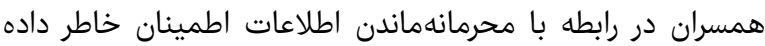

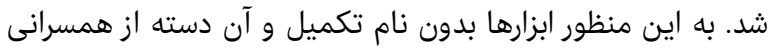

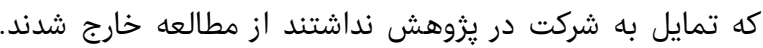

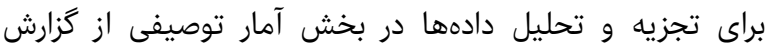

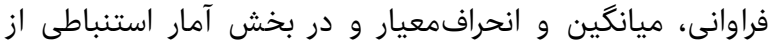

مطلوبى برخوردار خواهند بود[28]. همجنين ناميدى بر سلامتى و

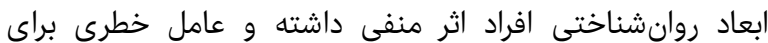

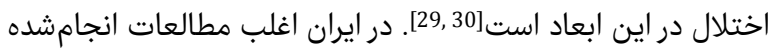

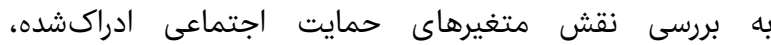

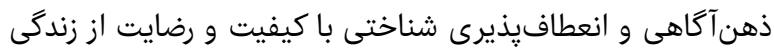

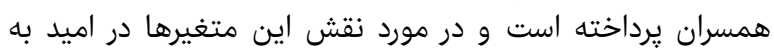
زندگى همسران يزوهشى صوراخنه است ورت نكرفته است.

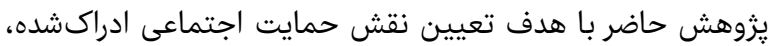

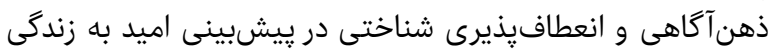
همسران جانبازان انجام شدان

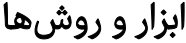

مطالعه توصيفى - همبستخى حاضر در سال هوسا در شهر اردبيل

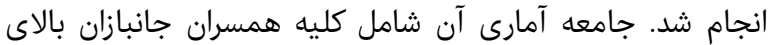

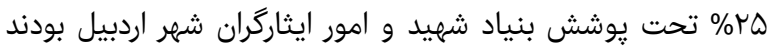

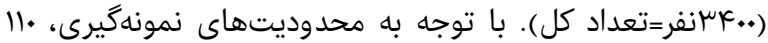

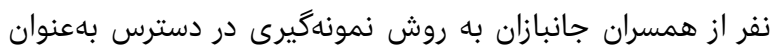

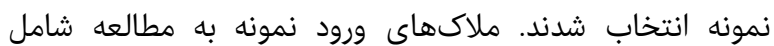

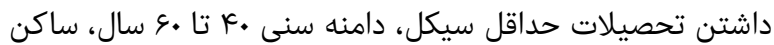

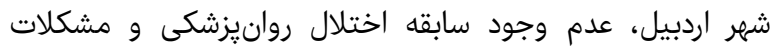

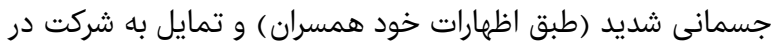

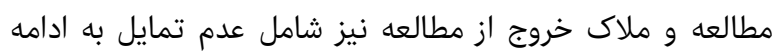

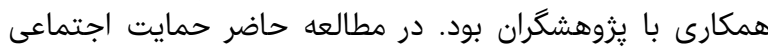

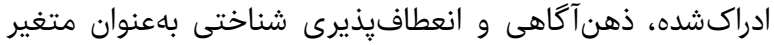

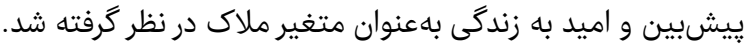

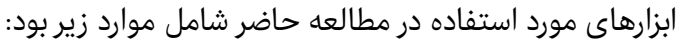

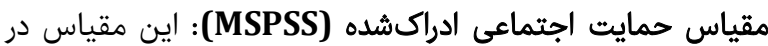

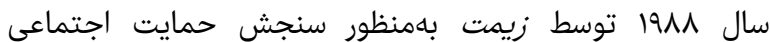

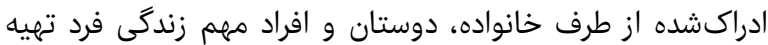

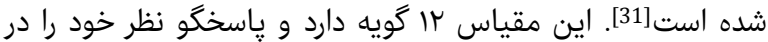

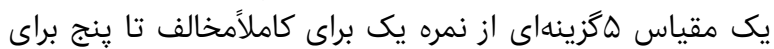

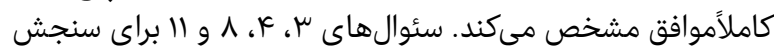

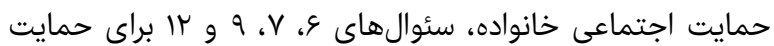

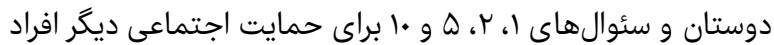

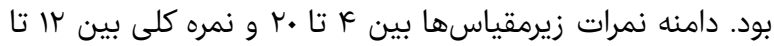

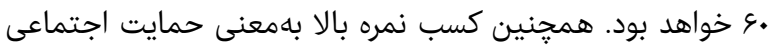

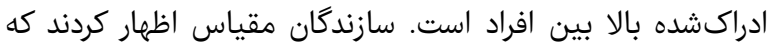

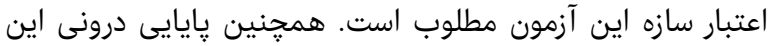

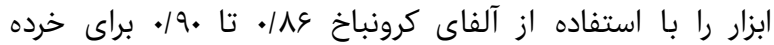

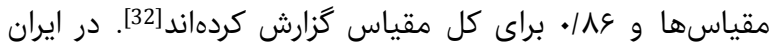

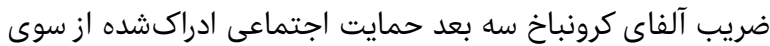

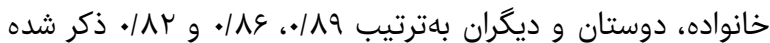

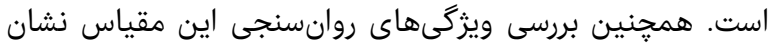

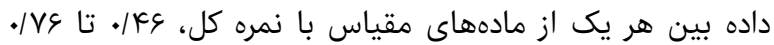

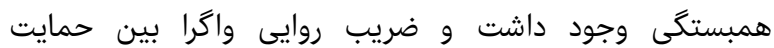

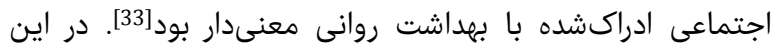

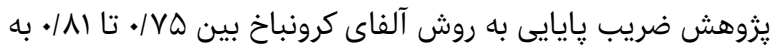

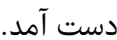
مقياس ذهنآكاهى (MASS): اين ابزار در سال س..ب توسط براون

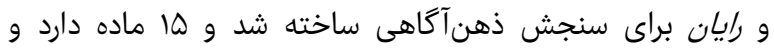

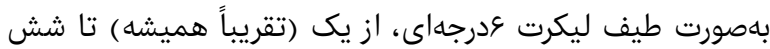

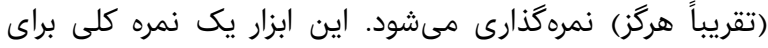


نتايج نشان داد كه بين حمايت اجتماعى ادراكشده و اميد به

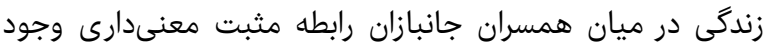

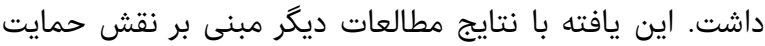

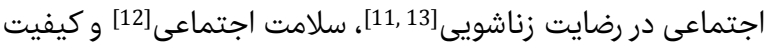

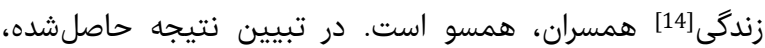

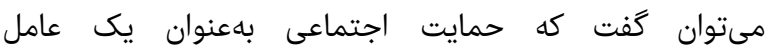

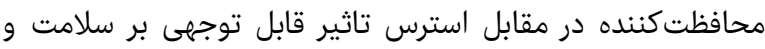

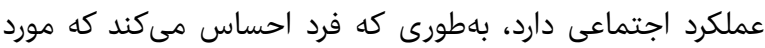

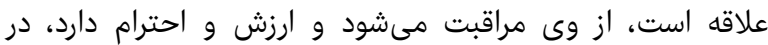

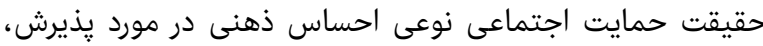

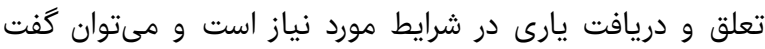

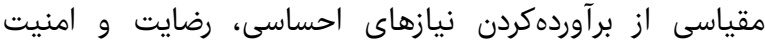

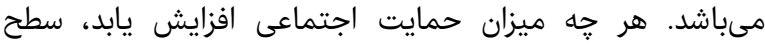

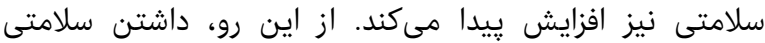

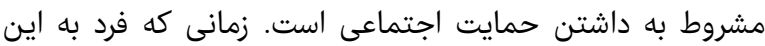

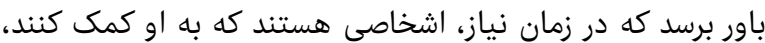

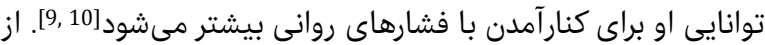

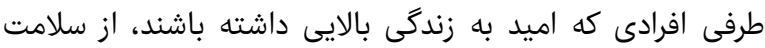

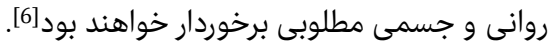

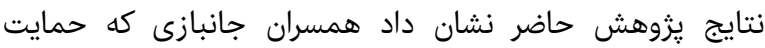

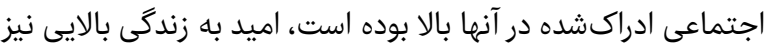

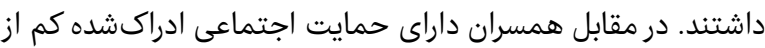

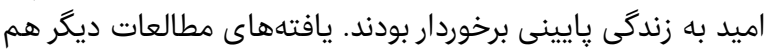

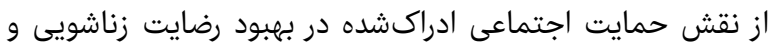

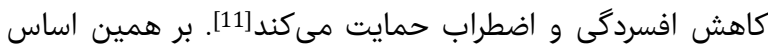

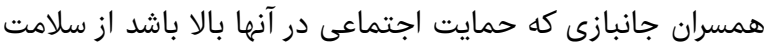

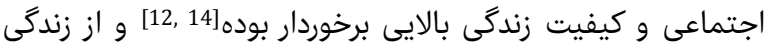

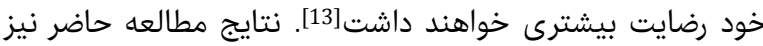

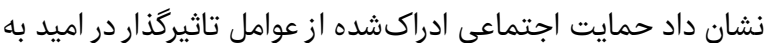

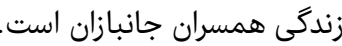

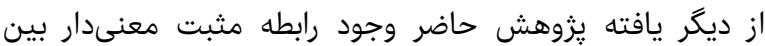

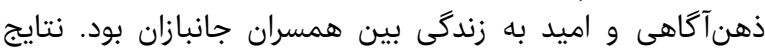

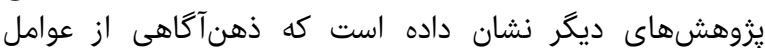
تاثيرگذار در بهبود سلامت

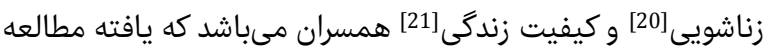

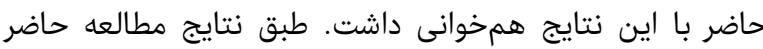

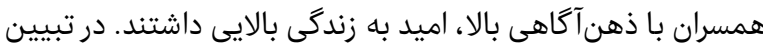

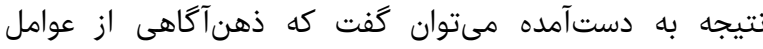

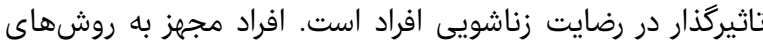

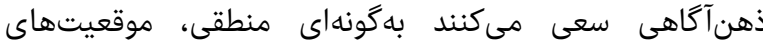

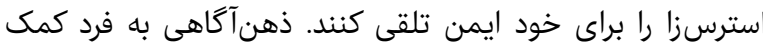

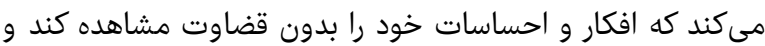

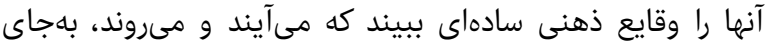

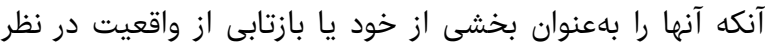

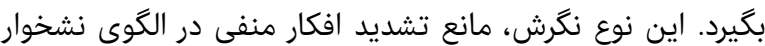

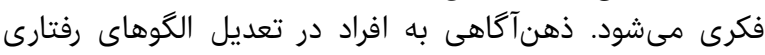

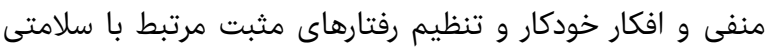

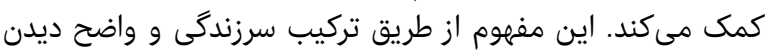

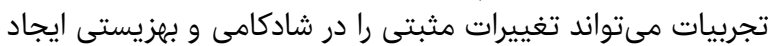

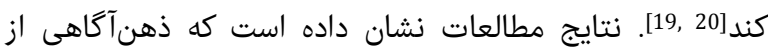

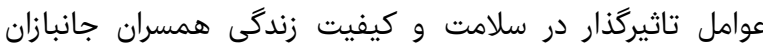

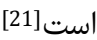

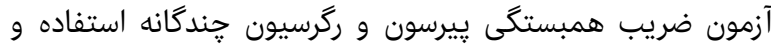

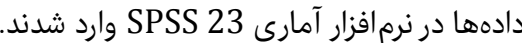

يافتهها

ميانگين آمارى سن شركت كنندگان

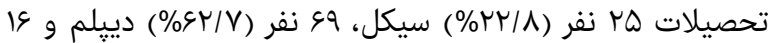

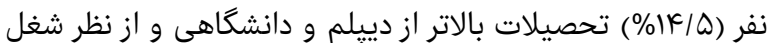

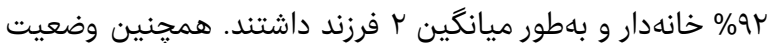

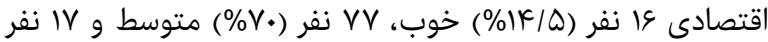

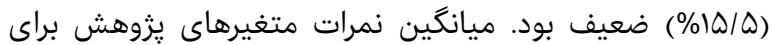

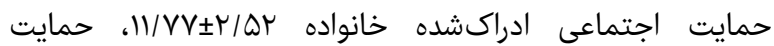

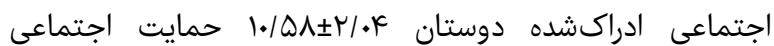

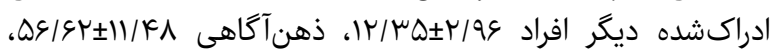

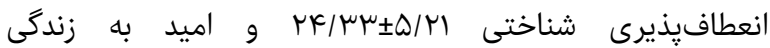
|

بين ذهن آكاهى با حمايت اجتماعى دست ادراكشده از سوى خانواده (r=/Kr)

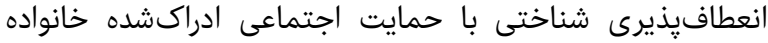
معن (r=/KV)

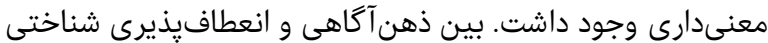
(r=-/FQ)

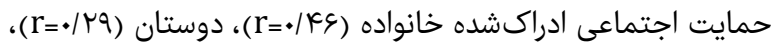

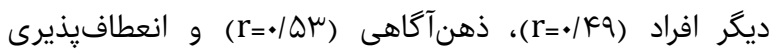

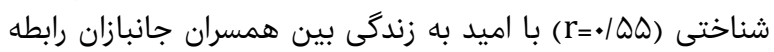

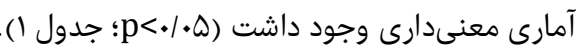

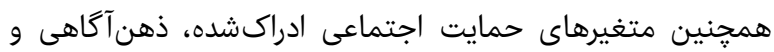

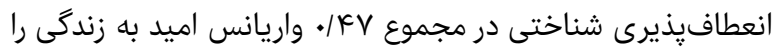

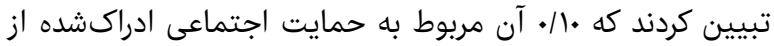

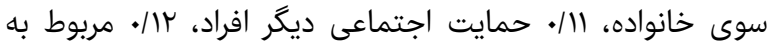

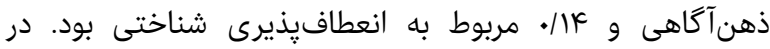

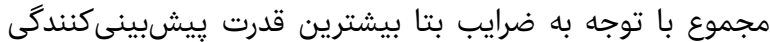

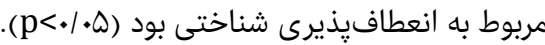
جدول () ماتريس همبستخى متغيرهاي يزوهش

\begin{tabular}{|c|c|c|c|c|c|}
\hline \multirow{2}{*}{\multicolumn{3}{|c|}{$\mu$}} & $r$ & 1 & 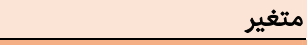 \\
\hline & & & & 1 & 1- حمايت اجتماعى خانواده \\
\hline & & & 1 & . & r- حمايت اجتماعى دوستان \\
\hline & & 1 & س & .101 & ץ- حمايت اجتماعى ديكر افراد \\
\hline & 1 &.$/ \mu q$ &.$/ T V$ & . $/ 4 r$ & F - ذهن آكاهى \\
\hline 1 &.$/ F \Delta$ & $\cdot / \mu$ &.$|\mu|$ & $\cdot / k V$ & هـ - انعطافيذيرى شناختى \\
\hline $1 \cdot 100$ & $\cdot / \Delta \mu$ &.$/ k q$ &.$/ 49$ &.$/ 44$ & צ- اميد به زندگى \\
\hline
\end{tabular}

بحث

يزوهش حاضر با هدف تعيين نقش حمايت اجتماعى ادراكشده،

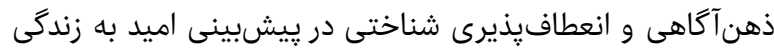

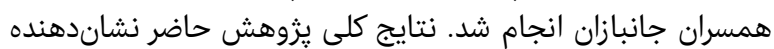

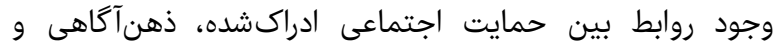

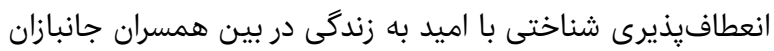

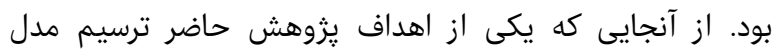

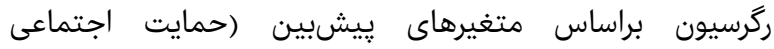

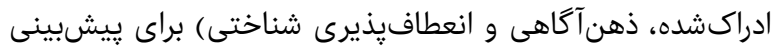

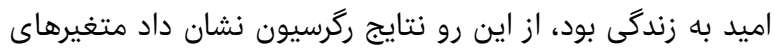

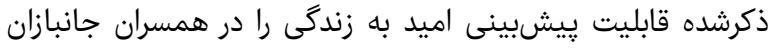

دارند. 
1- Mohseni M, Farnia M, Taghva A, Dehghan Manshadi Z, Rezaeifard A. Comparison of quality of life, depression, stress, anxiety and family structure in veteran's families with post-traumatic stress disorder (PTSD) patients and normal families. J War Public Health. 2014;6(5):207-14. [Persian]

2- Yambo TW, Johnson ME, Delaney KR, Hamilton R, Miller AM, York JA. Experiences of military spouses of veterans with combat-related posttraumatic stress disorder. J Nurs Scholarsh. 2016;48(6):543-51.

3- Moradi A, Ebrahimzadeh MH, Soroush MR. Quality of life of caregiver spouses of veterans with bilateral lower extremity amputations. Trauma Mon. 2015;20(1):e21891.

4- Kianpoor M, Rahmanian P, Mojahed A, Amouchie. Secondary traumatic stress, dissociative and somatization symptoms in spouses of veterans with PTSD in Zahedan, Iran. Electron Physician. 2017;9(4):4202-6.

5- Tsai J, Harpaz-Rotem I, Pietrzak RH, Southwick SM. The role of coping, resilience, and social support in mediating the relation between PTSD and social functioning in veterans returning from Iraq and Afghanistan. Psychiatry. 2012;75(2):135-49.

6- Kamari Sanghoriabadi S, Fathabadi J. The role of religious orientation and life expectancy in life satisfaction. J Mod Psychol Res. 2013;7(28):159-78. [Persian]

7- Najafi M. The role of hope, loneliness feeling and their components in prediction of elders' life satisfaction. J Couns Res. 2013;12(47):99-114. [Persian]

8- Williams AM, Cano A. Spousal mindfulness and social support in couples with chronic pain. Clin J Pain. 2014;30(6):528-35.

9- Sedighi Arfaee F, Hesampour F. The relationship between mindfulness and perceived social support and mental health in mothers of children with intellectual disability. Q J Soc Work. 2015;4(4):42-52. [Persian].

10- Dehle C, Landers JE. You can't always get what you want, but can you get what you need? Personality traits and social support in marriage. J Soc Clin Psychol. 2005;24(7):1051-76.

11- Maliszewska K, Świątkowska-Freund M, Bidzan M, Preis K. Relationship, social support and personality as psychosocial determinants of the risk for postpartum blues. Ginekol Pol. 2016;87(6):442-7.

12- Saleh S, ZahediAsl M. Correlation of social support with social health of psychiatry veterans wives. Iran J War Public Health. 2014;6(5):201-6. [Persian]

13- Sahraian K, Jafarzadeh F, Poursamar SL. The relationship between social support and marital satisfaction among infertile women based on their infertility factor. J Nurs Midwifery Urmia Univ Med Sci. 2015;12(12):1104-9. [Persian]

14- Hojjati H, Ebadi A, Zarea K, Akoundzadeh G, Alostani J, Nouhi E. Relationship between social support and quality of life dimensions of spouses of veterans with posttraumatic stress disorderrans of war. Mil Caring Sci. 2017;(4)2:87-94. [Persian]

15- Frewen PA, Evans EM, Maraj N, Dozois DJA, Partridge K. Letting go: Mindfulness and negative automatic thinking. Cognit Ther Res. 2008;32(6):758-74.

16- Walker LH, Colosimo K. Mindfulness, self-

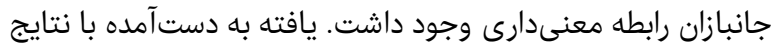

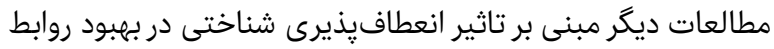

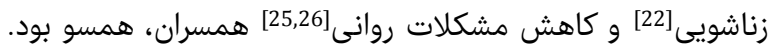

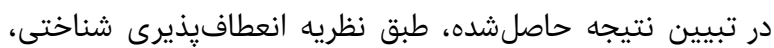

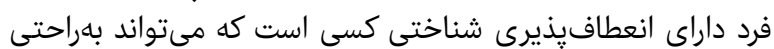

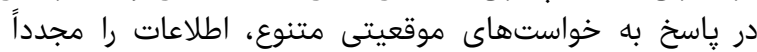

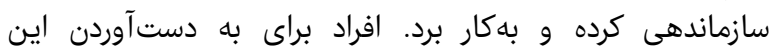

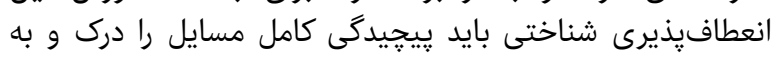

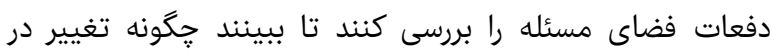

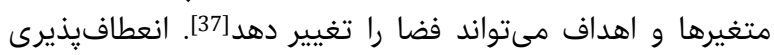

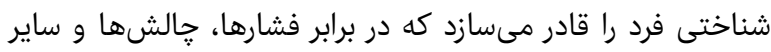

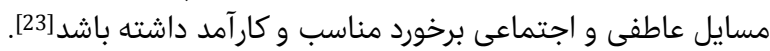

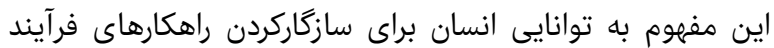

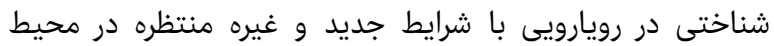

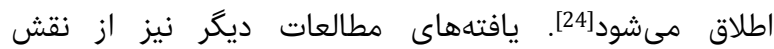

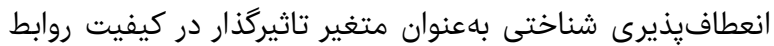

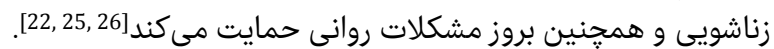

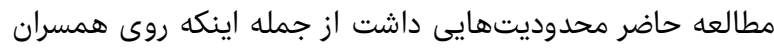

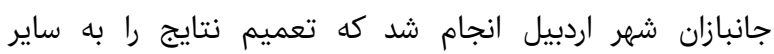

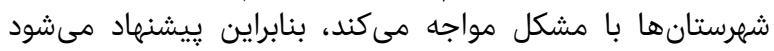

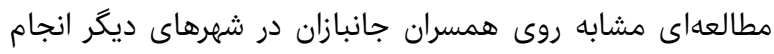

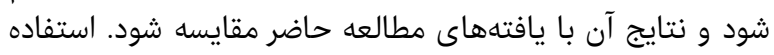

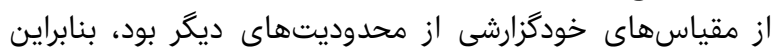

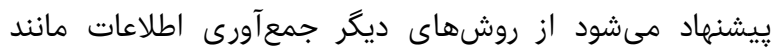

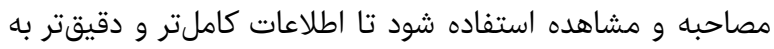

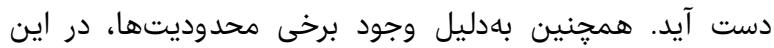

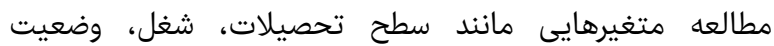

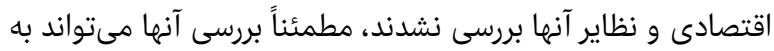

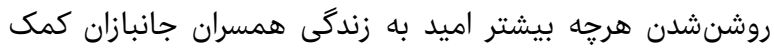

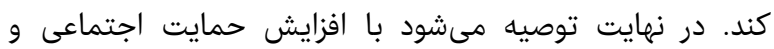

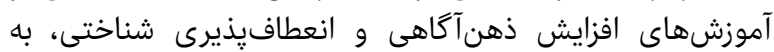
همسران در بهبود اميد به زندزى كمكى شود.

نتيجه گيرى

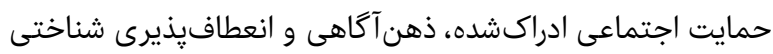

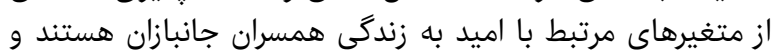

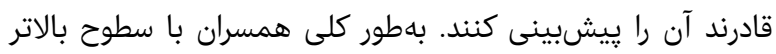

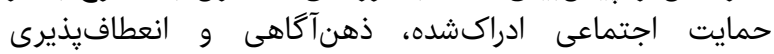

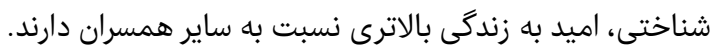

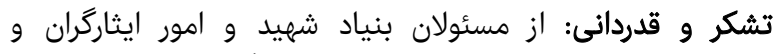

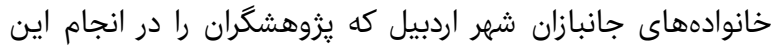

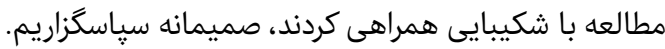

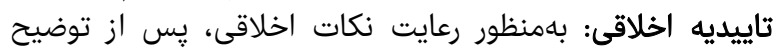

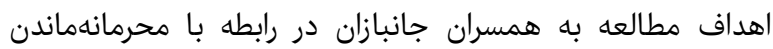
اطلاعات اطمينان خاطر داده شمان شدان

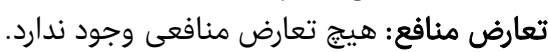

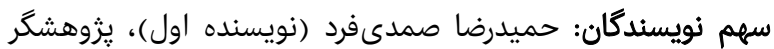

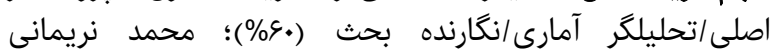

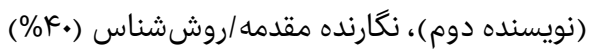


27. [Persian]

28- Nasiri HA, Joukar B. The relationship between life's meaningfulness, hope, happiness, life satisfaction and depression. Women Dev Polit. 2008;6(2):157-76. [Persian]

29- Shorey HS, Snyder CR, Yang X, Lewin MR. The role of hope as a mediator in recollected parenting, adult attachment and mental health. J Soc Clin Psychol. 2003;22(6):685-715.

30- Sadoughi M, Mehrzad V, Mohammad Salehi Z. The relationship of optimism and hope with depression and anxiety among women with breast cancer. J Nurs Res. 2017;12(2);16-21. [Persian]

31- Zimet GD, Dahlem NW, Zimet SG, Farley GK. The multidimensional scale of perceived social support. J Personal Assess. 1988;52(1):30-41.

32- Bruwer B, Emsley R, Kidd M, Lochner C, Seedat S. Psychometric properties of the multidimensional scale of perceived social support in youth. Compr Psychiatry. 2008;49(2):195-201.

33- Salimi A, Jokar B, Nikpoor R. Internet and communication: Perceived social support and Loneliness as antecedent variables. Psychol Stud. 2009;5(3):81-102. [Persian].

34- Ryan RM, Brown KW. Why we don't need selfesteem: On fundamental need, contingent love and mindfulness. Psychol Inq. 2003;14(1):71-6.

35- Ghorbani N, Watson PJ, Weathington BL. Mindfulness in Iran and the United States: Cross-cultural structural complexity and parallel relationships with psychological adjustment. Curr Psychol. 2009;28(6):211-24.

36- Dennis JP, Van Der Wal JS. The cognitive flexibility inventory: Instrument development and estimates of reliability and validity. Cogn Ther Res. 2010;34(3):24153.

37- Soltani E, Shareh H, Bahrainian SA, Farmani A. The mediating role of cognitive flexibility in correlation of coping styles and resilience with depression. Pejouhandeh. 2013;18(2):88-96. [Persian]

38- Snyder CR, Harris C, Anderson JR, Holleran SA, Irving LM, Sigmon ST, et al. The will and the ways: Development and validation of an individual-differences measure of hope. J Pers Soc Psychol. 1991;60(4):570-85. 39- Samadifard HR. Prediction of life expectancy of spouses based of meta-cognitive belief and cognitive fusion. J Res Clin Psychol Counsel. 2017;6(2):48-62. [Persian] compassion and happiness in non-meditators: A theoretical and empirical examination. Personal Individ Differ. 2011;50(2):222-7.

17- Wang Y, Xu W, Luo F. Emotional resilience mediates the relationship between mindfulness and emotion. Psychol Rep. 2016;118(3):725-36.

18- Dahm K, Meyer EC, Neff K, Kimbrel NA, Gulliver SB, Morissette SB. Mindfulness, self-compassion, posttraumatic stress disorder symptoms, and functional disability in U.S. Iraq and Afghanistan war veterans. J Trauma Stress. 2015;28(5):460-4.

19- Schellekens MPJ, Karremans JC, Van der Drift MA, Molema J, Van den Hurk DGM, Prins JB, et al. Are mindfulness and self-compassion related to psychological distress and communication in couples facing lung cancer? A dyadic approach. Mindfulness. 2017;8(2):325-36.

20- Madani Y, Hojati S. The effect of mindfulness-based cognitive therapy on marital satisfaction and quality of life in couples. Appl Psychol Res Q. 2015;6(2):39-60. [Persian]

21- Zarnaghash M. The effectiveness of mindfulnessbased stress reduction on quality of life and resiliency of veterans spouses in Ahvaz. J Psychol Models Methods. 2016;7(23):83-98. [Persian]

22- SedaghatKhah A, BehzadiPoor S. Predicting the quality of marital relationship on the base of relationship beliefs, mindfulness and psychological flexibility. Q J Woman Soc. 2017;8(30):57-77. [Persian]

23- Dajani DR, Uddin LQ. Demystifying cognitive flexibility: Implications for clinical and developmental neuroscience. Trends Neurosci. 2015;38(9):571-8.

24- Rostami CH, Jahangirlu A, Sohrabi A, Ahmadian H. The role of cognitive flexibility and mindfulness in predicting student Procrastination. Zanko J Med Sci. 2016;17(53):50-61. [Persian]

25- Lee JK, Orsillo SM. Investigating cognitive flexibility as a potential mechanism of mindfulness in generalized anxiety disorder. J Behav Ther Exp Psychiatry. 2014;45(1):208-16.

26- Keith J, Velezmoro R, O'Brien C. Correlates of cognitive flexibility in veterans seeking treatment for posttraumatic stress disorder. J Nerv Ment Dis. 2015;203(4):287-93.

27- Eskandari H, Pajouhinia Sh, Abavisan Y. Explanation of psychological problems based on cognitive flexibility and self differentiation. Shefaye Khatam. 2016;4(3):18- 